\title{
Janus Particles with Flower-Like Patches Prepared by Shadow Sphere
}

\section{Lithography}

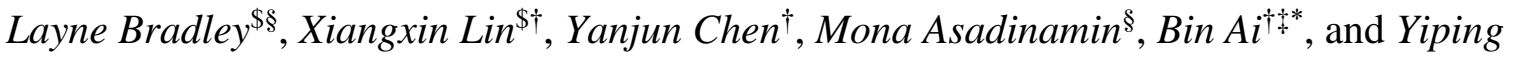

Zhao ${ }^{\S}$

${ }^{\dagger}$ School of Microelectronics and Communication Engineering, Chongqing University,

Chongqing 400044, P.R. China

Chongqing Key Laboratory of Bio perception \& Intelligent Information Processing,

Chongqing 400044, P.R. China

${ }^{\S}$ Department of Physics and Astronomy, University of Georgia, Athens, Georgia 30602, USA

\$the authors contributed equally to this work.

E-mail: binai@cqu.edu.cn

\section{Table of Contents}

Section S1. Mechanism of the Prediction Program

Section S2. User Guide of the Prediction Program

Section S3. FDTD Calculation of $E$-field Distribution.

Section S4. SEM Images of Patches on $500 \mathrm{~nm}$ Spheres

Section S5. Optical Properties of Patches on $2 \mu \mathrm{m}$ Spheres

Section S6. Calculation of Roundedness

Section S7. Transmission Spectra 


\section{Section S1. Mechanism of the Prediction Program}

A simple simulation is used to estimate the thickness distribution of the patchy structures. As illustrated in Figure S1a, an array of close-packed beads with diameter $D=$ $500 \mathrm{~nm}$ is in the $\mathrm{x}-\mathrm{y}$ plane. A uniform vapor flux approaches the array of beads in direction $\hat{l}$ $\left(\theta_{0}, \varphi_{0}\right)$. In order to calculate the thickness distribution on bead $O$, the nearest 36 beads are taken into account, which potentially affect the deposition on bead $O$. As shown in Figures $\mathrm{S} 1 \mathrm{~b}$ and $\mathrm{S} 1 \mathrm{c}$, the surface of bead $O$ is split into small surface elements. Each element is denoted as the coordinate of its center $(\theta, \varphi)$, where $\theta=i \cdot \Delta \theta, \varphi=j \cdot \Delta \varphi, \Delta \theta=\Delta \varphi=0.5^{\circ}, i=0$, $1, \ldots 360, j=0,1, \ldots 720$.
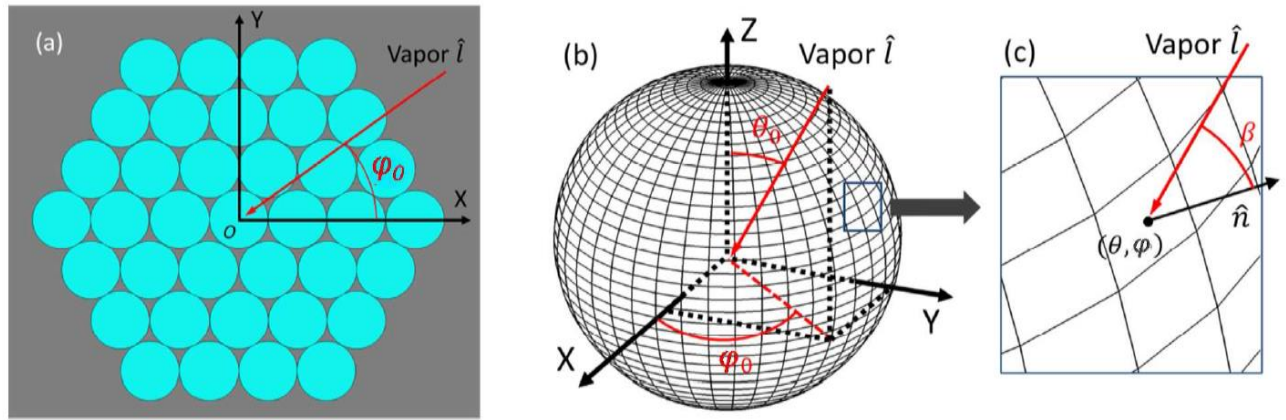

Figure S1. (a) Sketch of vapor deposition on an array of beads, (b) the surface of bead $O$ split into numbers of surface elements, (c) a surface element on bead $O$.

Our simulation is based on three assumptions:

1. Only the shadowing effect and material accumulation are considered. Other physical process, such as surface diffusion, is neglected.

2. The deposition at different surface elements happens simultaneously as long as they are directly exposed to vapor.

3. The as-deposited film is non-porous and is uniform within each surface element.

In our simulation of patchy structures, there are three simulation steps. A $120^{\circ}$ azimuthal 
rotation of $\hat{l}$ happens at the end of each step. In each simulation step, the thickness at each surface element $h(\theta, \varphi)$ is updated,

$$
h_{i+1}(\theta, \varphi)=h_{i}(\theta, \varphi)+\Delta h
$$

In order to calculate the change of thickness $\Delta h$, we need to first determine whether point $(\theta, \varphi)$ is directly exposed to the vapor flux. If point $(\theta, \varphi)$ is in the shadow of other structures, then $\Delta h=0$. For the uniform condition, the shadowed area does not take into account previously deposited material, i.e., the surface profile of the template remains constant. For the $\mathrm{CW}$ and $\mathrm{CCW}$ simulations, previously deposited material on neighboring beads will contribute to the shadow because the surface profile is updated at each step. These different conditions are achieved by considering the following effects. If $(\theta, \varphi)$ can receive vapor, then $\Delta h$ can be expressed as,

$$
\Delta h=\frac{\Delta m}{\rho \cdot S(\theta, \varphi)},
$$

where $\Delta m$ is the mass of material deposited on surface element $(\theta, \varphi)$ within time $\Delta t, \rho$ is the density of the material, and $S(\theta, \varphi)$ is the area of surface element $(\theta, \varphi)$. The vapor flux $\Phi$ is defined as,

$$
\Phi=\frac{\Delta m}{\Delta t \cdot S_{n}}=\text { const }
$$

where $S_{\mathrm{n}}$ is the projection of $S(\theta, \varphi)$ onto the plane perpendicular to the vapor flux $\hat{l}$. With $\beta$ defined as the angle between $\hat{l}$ and the surface normal vector $\hat{n}, S_{\mathrm{n}}$ can be expressed as,

$$
S_{N}=S(\theta, \varphi) \cos \beta
$$

by combining Equations S2-S4, we obtain the final expression of $\Delta h$,

$$
\Delta h=\frac{\Phi}{\rho} \Delta t \cos \beta,
$$

In each step, we set $\frac{\Phi}{\rho} \Delta t$ as $15 \mathrm{~nm}$. 


\section{Section S2. User Guide of the Prediction Program}

The prediction program can be available at website: http://ailabcqu.com/GLAD? I=en, Its user interface is as shown in Figure S2. The user guide is as follows:

1. Set 'sphere radius', 'distance between spheres' (period), 'incident angle' $\theta$, and 'deposition' thickness.

2. Set azimuthal rotation angle $\varphi$ at 'OAD (oblique angle deposition) Phi'.

3. Click on 'OAD' and the program will run.

4. Click on 'Plot_Sub' to get patterns on substrate. Click on 'Plot_Bead' to get patchy patterns on spheres.

5. Repeat step 1 - 4 to get multilayer patterns. Hit 'Initialization' to clear previous calculations.

6. Set parameters in GLAD (glancing angle deposition) to run multiple calculations at different rotation angles.

7. To get the original data for further operation, please click 'Save sub as txt' and 'Save bead as txt'.

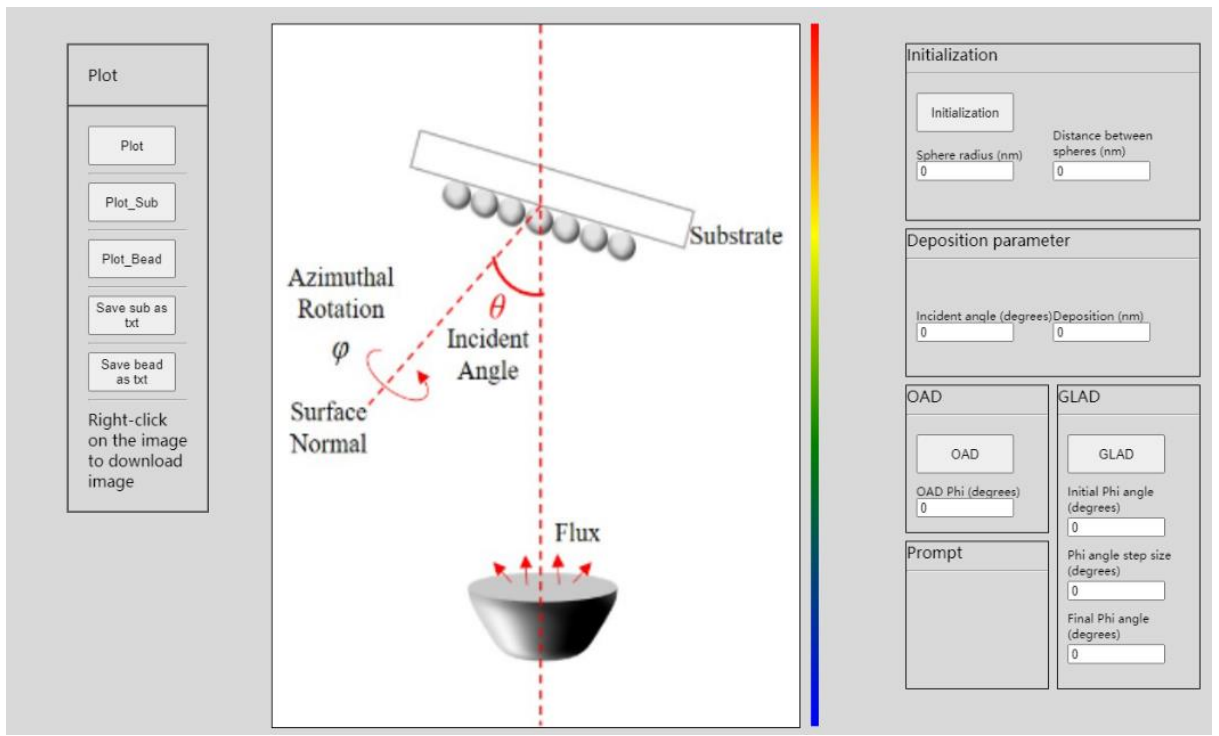

Figure S2. The user interface of the prediction program. 


\section{Section S3. FDTD Calculation of $E$-field Distribution.}

A commercialized software package, finite-different time-domain (FDTD) Solutions (Lumerical Solutions, Inc. Canada), was used to calculate the local electric field ( $E$-field) distribution of a single patch extracted from the MATLAB predicted structures. The patch was fabricated on a $500 \mathrm{~nm}$ sphere with $n=5, \theta=75^{\circ}$, and $\varphi_{0}=0^{\circ}$. A FDTD region with perfect metal layer (PML) boundary conditions in three dimensions and total-field scatteredfield source with polarization along the $x$ axis were used. Override $3 \mathrm{~nm}$ mesh was applied for the entire source region. The $E$-field distribution data was collected by a monitor of threedimension (3D) 'frequency domain field profile' at wavelength $\lambda=633 \mathrm{~nm}$ which is the one of the most common laser wavelengths for surface enhanced Raman scattering (SERS). As shown in Figure S3, other than the dipole resonance of the shell, $E$-fields can be clearly enhanced along the tortuous edge with sharp tips and small gaps, which would benefit the plasmonic-related applications, such as sensors, imaging, and surface enhanced Raman scattering (SERS). The smooth patch surface does not show enhanced $E$-fields. The $E$-fields distributed near the equator is generated by the scattering of the PS spheres.

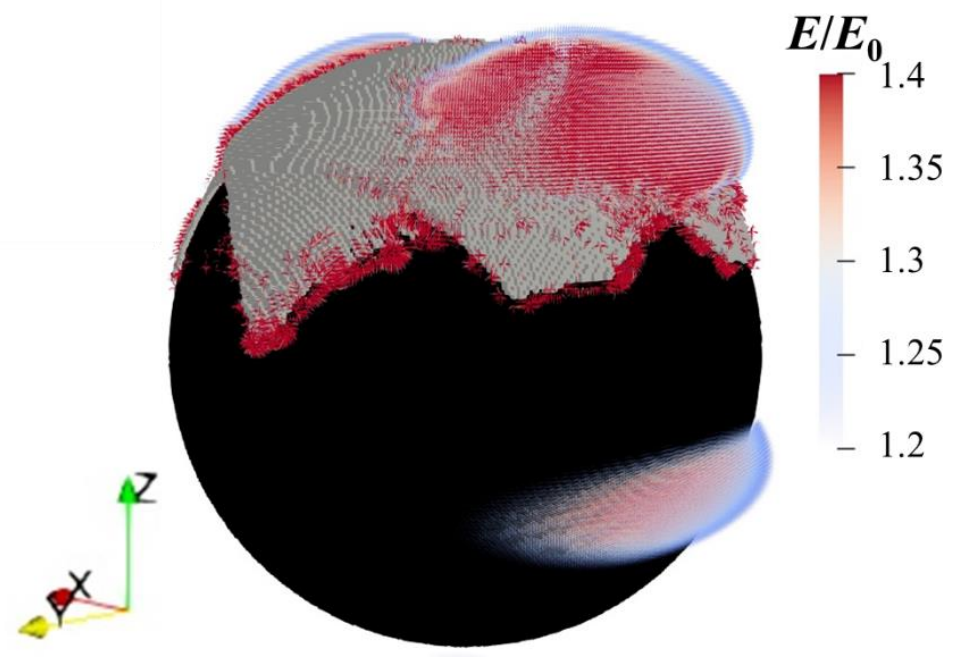

Figure S3. FDTD calculation of the $E$-field distribution of the patch on a $500 \mathrm{~nm}$ sphere 
performed at $n=5, \theta=75^{\circ}$, and $\varphi_{0}=0^{\circ}$. The wavelength for the calculation is at $633 \mathrm{~nm}$. The black and white area are the sphere and patch, respectively. 
Section S4. SEM Images of Patches on $500 \mathrm{~nm}$ Spheres

$\mathbf{a}$
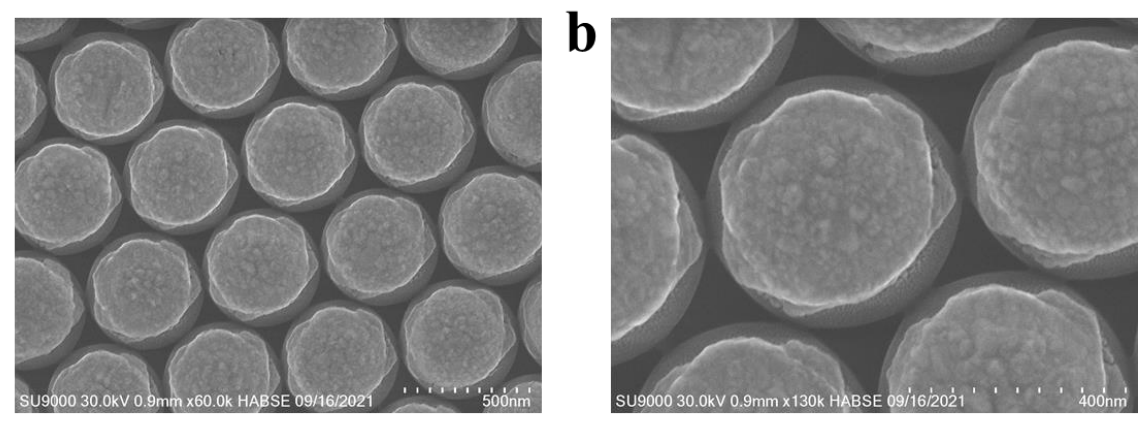

c


Figure S4. Representative (a) SEM image and (b) zoom-in SEM image of patches formed on $500 \mathrm{~nm}$ spheres from depositions performed at $\theta=75^{\circ}$ and $n=5$. Representative (c) SEM image and (d) zoom-in SEM image of the dispersed JPs with the patches formed on $500 \mathrm{~nm}$ spheres from depositions performed at $\theta=75^{\circ}$ and $n=5$. 
Section S5. Optical Properties of Patches on $2 \mu \mathrm{m}$ Spheres
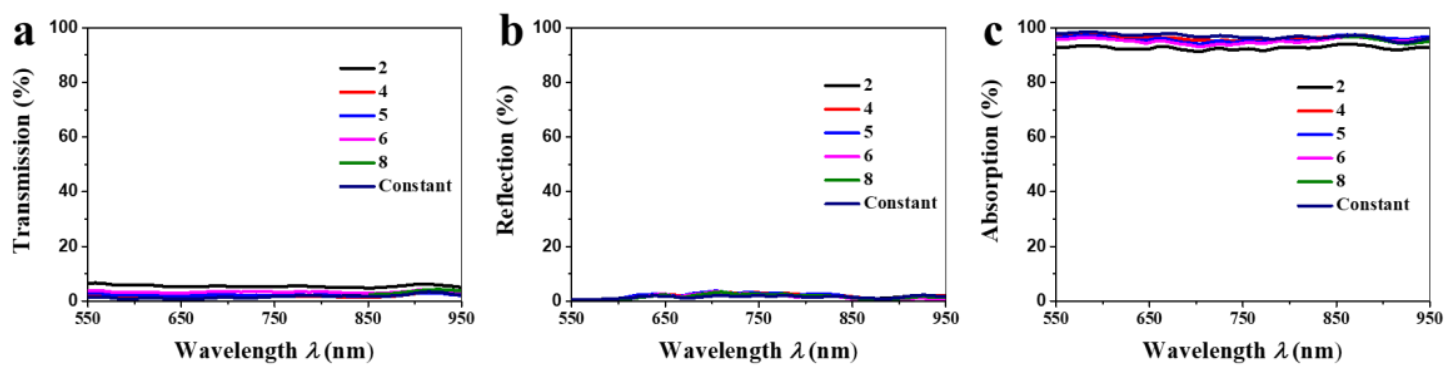

Figure S5. (a) Transmission, (b) reflection, and (c) absorption spectra of the patches on $2 \mu \mathrm{m}$ spheres at $\theta=75^{\circ}, n=2,4,5,6,8$, and $\infty$ (constant rotation). These spectra are averaged from 9 domains. 


\section{Section S6. Calculation of Roundedness}

Outlines of the sphere and patch can be sketched by the software ImageJ (Figure S6a), which are extracted as shown in Figure S6b. The $S$ in Figure S6b is the maximum radius for the deposition, which normally equals the sphere radius. The distance from every point at the edge to the center can be calculated as $s_{i}$. Figure S6c shows a set of data of the patches performed at $\theta=75^{\circ}, n=5$, and varied $\varphi_{\mathrm{o}}$. The patch patterns are similar, which can be supported by the small errors in Figure $\mathbf{5 b}$ and Figure $\mathbf{9 b .}$
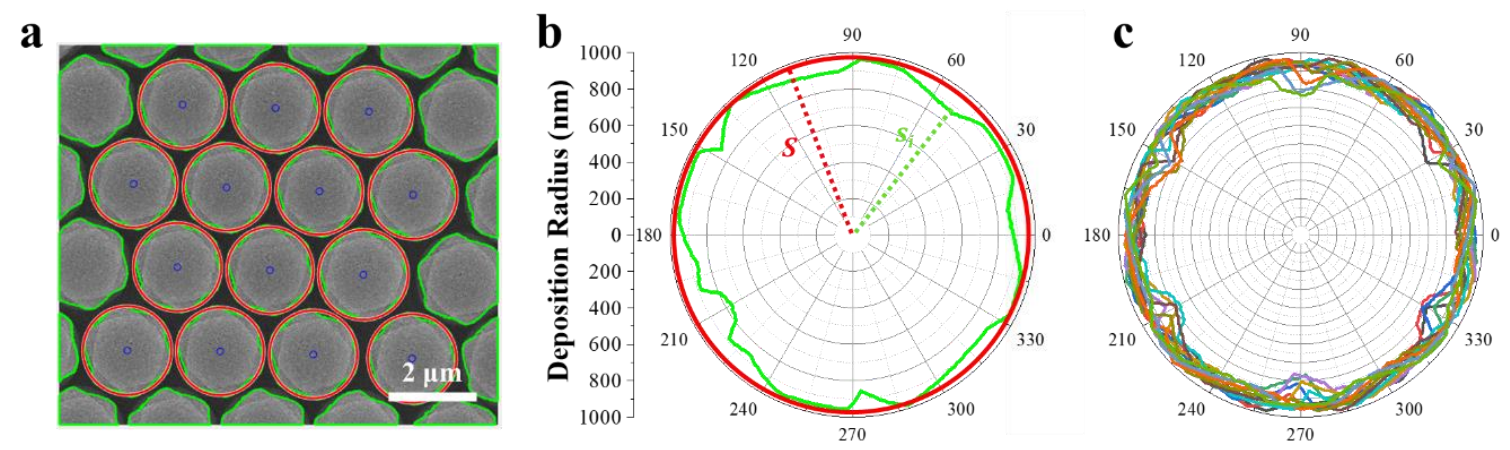

Figure S6. (a) SEM image of the patches formed on $2 \mu \mathrm{m}$ spheres from depositions performed at $\theta=75^{\circ}, n=5$, and $\varphi_{\mathrm{o}}=30^{\circ}$. The red and green curves show the outlines of the sphere and patch pattern. (b) Polar graph of the patch pattern from (a). $S$ is the maximum radius for the deposition (radius of the sphere) and $s_{i}$ is the distance from the center to the number $i$ point at the pattern edge. (c) Polar graph of the patches formed on $2 \mu \mathrm{m}$ spheres from depositions performed at $\theta=75^{\circ}, n=5$, and $\varphi_{\mathrm{o}}=0^{\circ}, 5^{\circ}, 10^{\circ}, 15^{\circ}, 20^{\circ}, 25^{\circ}, 30^{\circ}, 35^{\circ}$, $40^{\circ}, 45^{\circ}, 50^{\circ}$, and $55^{\circ}$. 


\section{Section S7. Transmission Spectra}
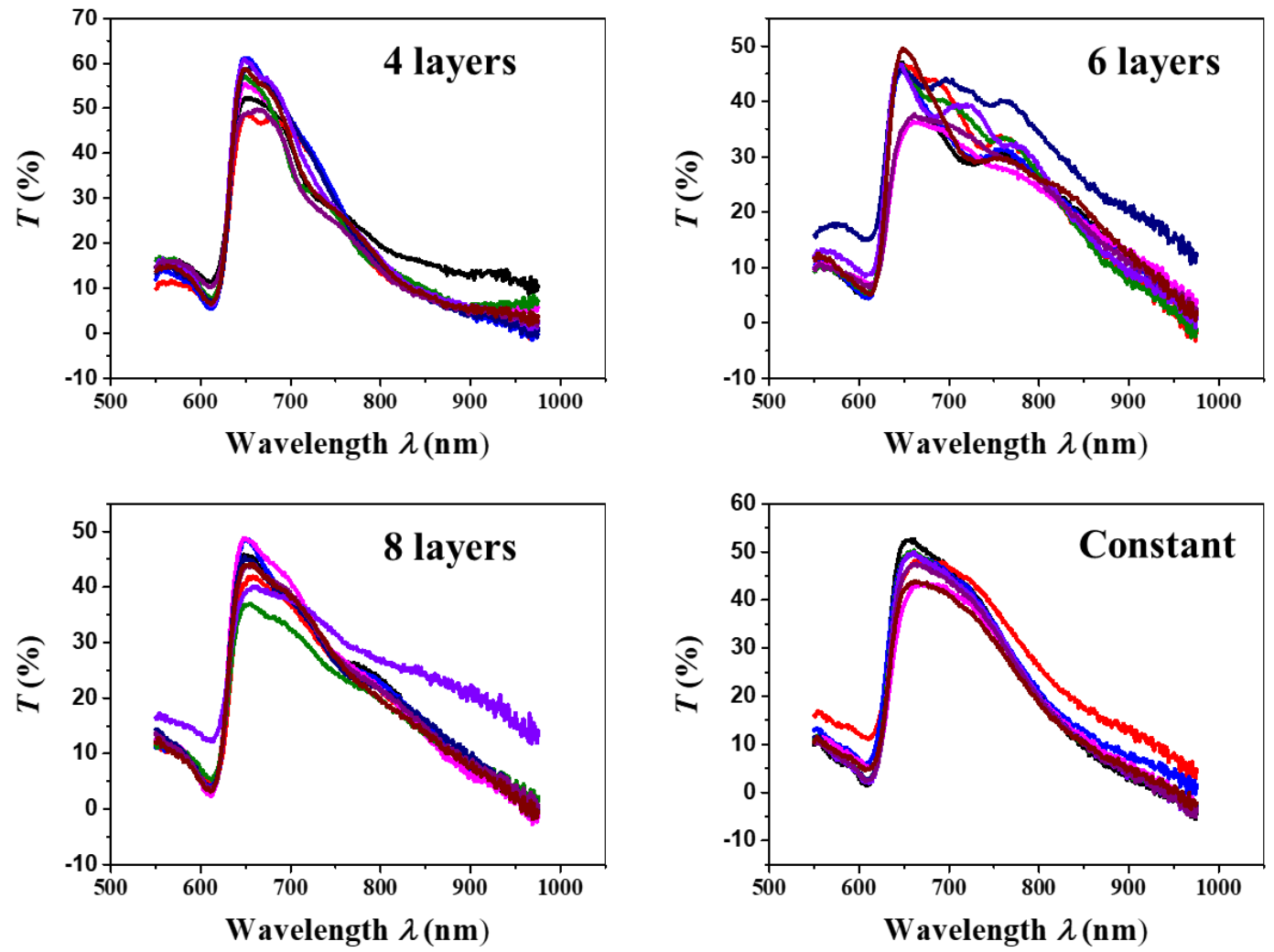

Figure S7. Transmission spectra of patches formed on $500 \mathrm{~nm}$ spheres from depositions performed at $\theta=75^{\circ}, n=4,6,8$, and $\infty$ (constant rotation). 9 domains were measured for each sample.
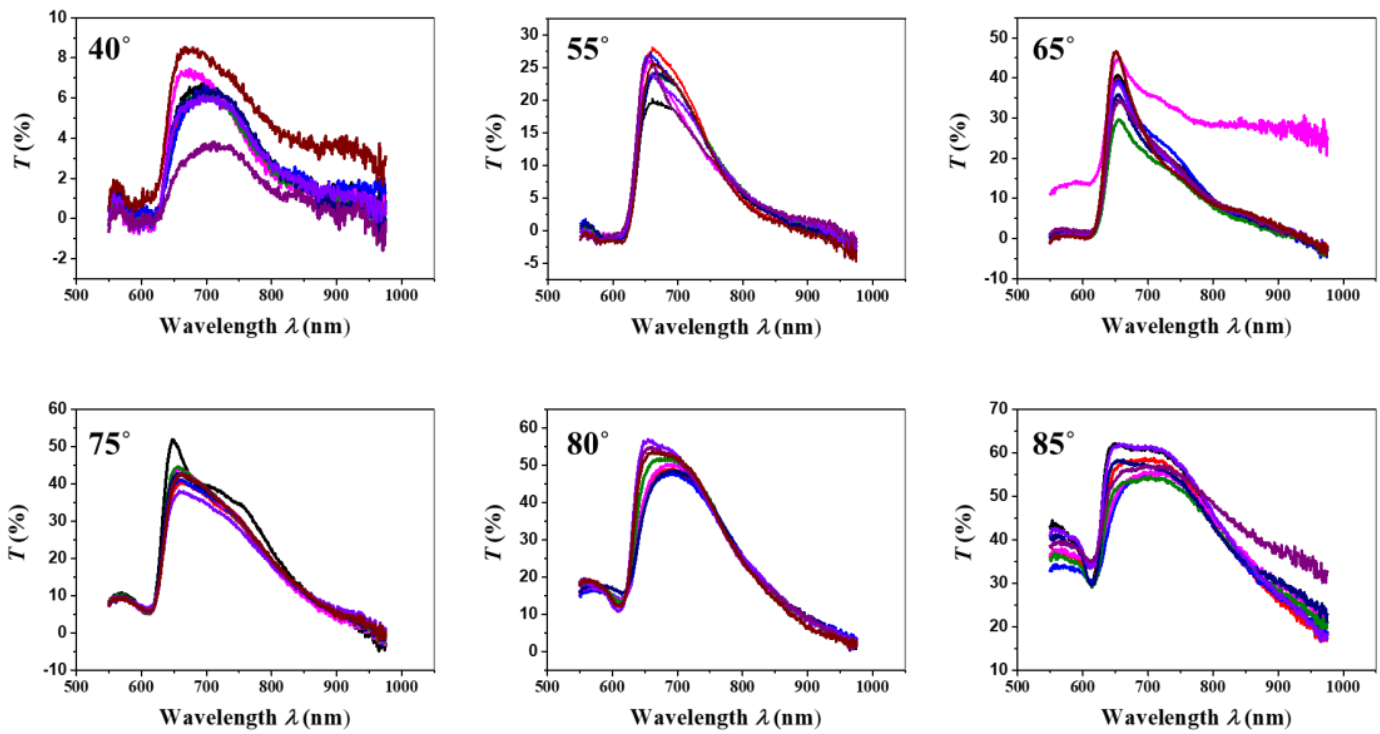

Figure S8. Transmission of patches formed on $500 \mathrm{~nm}$ spheres for depositions performed at $n=5, \theta=40^{\circ}, 55^{\circ}, 65^{\circ}, 75^{\circ}, 80^{\circ}$, and $85^{\circ} .9$ domains were measured for each sample. 